\title{
On Ascending Vickrey Auctions for Heterogeneous Objects*
}

\author{
Sven de Vries ${ }^{\dagger} \quad$ James Schummer ${ }^{\ddagger} \quad$ Rakesh V. Vohra ${ }^{\ddagger}$
}

August 23, 2005

\begin{abstract}
We construct an ascending auction for heterogeneous objects by applying a primal-dual algorithm to a linear program that represents the efficient-allocation problem for this setting. The auction assigns personalized prices to bundles, and asks bidders to report their preferred bundles in each round. A bidder's prices are increased when he belongs to a "minimally undersupplied" set of bidders. This concept generalizes the notion of "overdemanded" sets of objects introduced by Demange et al. (1986) for the one-to-one assignment problem.

Under a submodularity condition, the auction implements the Vickrey-Clarke-Groves outcome; we show that this type of condition is somewhat necessary to do so. When classifying the ascending-auction literature in terms of their underlying algorithms, our auction fills a gap in that literature. We relate our results to various ascending auctions in the literature.
\end{abstract}

Keywords: Vickrey auctions, multi-item auctions, combinatorial auctions, duality, primal-dual algorithm, subgradient algorithm

*We gratefully acknowledge invaluable discussions with Sushil Bikhchandani, and thank Larry Ausubel for comments. An Associate Editor's comments on exposition were particularly helpful. The research of the third author was supported in part by NSF grant ITR IIS-0121678.

†Zentrum Mathematik, TU München, D-85747 Garching bei München, Germany.

‡Department of Managerial Economics and Decision Sciences, Kellogg School of Management, Northwestern University, Evanston IL 60208. 


\section{Introduction}

Though much of auction theory has developed in the context of sealed-bid auctions, there are practical arguments (Cramton, 1998) favoring the use of "ascending" auctions. For example, such dynamic procedures may create more transparency in the auctioneer's methods, bidders may reveal less private information, communication and computation costs may be decreased, etc. These arguments are supported by the circumstantial evidence that the second-price (Vickrey) sealed-bid auction is unknown to many laypersons in that form, while its ascending version - the English auction-is commonly known.

While it is easy to see that the English auction dynamically implements the second-price auction of a single object, ascending counterparts may be less obvious in other environments. Indeed, previous works in the literature have derived such generalizations for special cases, as we discuss below. In this paper, we consider the general case in which an auctioneer wishes to sell a set of heterogeneous, indivisible objects to bidders with possibly non-additive valuations. We derive what we consider to be the appropriate generalization of the English auction ${ }^{1}$ for this model by using a method suggested by Bikhchandani et al. (2002). Specifically, we formulate the efficient object-assignment problem as a particular linear program. Afterwards, we examine an algorithm that is used to solve such linear programs, and show how it can be interpreted as an ascending auction.

\section{Generalizing the English Auction}

There are at least two natural ways in which the English auction can be generalized to other environments, depending on how one interprets the outcome of a second-price auction in the single-object case.

In one interpretation, the price paid by the winner can be viewed as a Walrasian price: when everyone is offered the object at this price, every bidder can make a purchase decision in a way such that the market "clears." That is, only one bidder wishes to consume the object. ${ }^{2}$ Under this interpretation, a generalization of the English auction should terminate in "minimal Walrasian prices" that support the efficient assignment of objects.

\footnotetext{
${ }^{1}$ It may be more accurate to say that we generalize the Japanese (button) auction.

${ }^{2}$ More technically, at most one bidder strictly wishes to, and at least one weakly wishes to. The second-price auction ends at the minimal such price.
} 
In the second, the price can be interpreted as the amount of surplus the winning bidder takes away from the other bidders by receiving the object. This more abstract interpretation leads to the general concept of the VickreyClarke-Groves (VCG) sealed-bid auction. In more general situations, a VCG auction makes efficient allocation decisions and charges bidders the amount by which they impose a surplus loss on the other bidders. Under this interpretation, a generalization of the English auction should terminate in "VCG prices" that support the efficient assignment.

While both generalizations involve efficient object-assignments, Walrasian and VCG payments typically differ in more general settings. In the assignment problem, however, they are equivalent. In this setting (involving heterogeneous objects, where bidders consume at most one object), Demange et al. (1986) construct an ascending auction which results in VCG (equivalently, minimal Walrasian) payments. In this auction, bidders declare which objects they prefer at current prices, and prices are increased on "overdemanded" sets of objects - objects which are outnumbered by the bidders who require them.

In contrast, even for the specialized setting in which all objects being sold are identical (i.e. homogeneous), Walrasian prices and VCG payments need not coincide. Ausubel (2004) has designed an ascending auction which results in VCG payments for this case. On the other hand, the ascending auction that produces minimal Walrasian prices for this case (under the assumption of sincere bidding) is the well known uniform-price auction.

To better understand the differences between the VCG and Walrasian interpretations, it is useful to think of an ascending auction as an algorithm which optimizes the use of the auctioneer's resources.

\section{A Systematic Approach}

In linear programming problems, the dual variable of a constraint represents the increase in value which could be obtained by relaxing that constraint. A bidder's profit in a VCG auction is the increase in "value" (of the objects) that can be attributed to that bidder's presence. Bikhchandani and Ostroy (2002) relate these two observations by formulating the efficient-assignment problem as a linear program whose dual variables yield the bidders' profits in a VCG auction (when a certain condition is satisfied).

We take this idea one step further by interpreting a primal-dual algorithmused to solve such linear programs - as an ascending auction for the heteroge- 
neous object case, when bidders may have non-additive valuation functions. This "recipe" for designing auctions, briefly outlined by Bikhchandani et al. (2002), is as follows.

1. Formulate the efficient-assignment problem as a linear program. By doing this appropriately (a là Bikhchandani and Ostroy, 2002) dual variables can be interpreted as the payments made by bidders and/or the surplus they receive.

2. Formulate a primal-dual algorithm for this linear program.

3. Interpret the algorithm as an auction.

We perform the first step in Section 2. The dual of our linear program provides bidder-specific prices for bundles of objects. In Section 3 we show how the primal-dual algorithm lends itself to an auction interpretation. In particular, it asks bidders to list the bundles of objects they demand at current prices, and then adjusts those prices accordingly. In Section 4 we discuss situations in which our generalization of the English auction in fact implements VCG payments.

It is natural to wonder whether this recipe can be used by replacing, in Step 2, the primal-dual algorithm with some other iterative algorithm used to solve linear programs. For example, a subgradient algorithm also works by adjusting dual variables; could it also yield an ascending auction? In Section 5 we show that a subgradient algorithm for this problem can be interpreted as the auction described by Ausubel and Milgrom (2002).

More generally, if one examines the special cases that have appeared in the literature, it appears that many of the well-known ascending auctions can be derived from either a primal-dual or subgradient algorithm. Table 1 categorizes such auctions, where the underlying algorithm operates on optimization problems whose variables correspond to prices. ${ }^{3}$

One difference between the two algorithms is the information they utilize at each iteration. In auction terms, a primal-dual algorithm requires a bidder to report his entire demand correspondence at each iteration, while a

\footnotetext{
${ }^{3}$ Notable exceptions to this particular classification are the works of Ausubel (2000) and Parkes and Ungar (2002). While those works can certainly be related to such algorithms (e.g. there is explicit use of primal-dual algorithms in the latter paper), they operate with the use of additional parameters (variables) which, in our view, cannot be interpreted as "ascending prices." See the end of Section 4.2.
} 


\begin{tabular}{lll}
\hline Environment & Primal-Dual & Subgradient \\
\hline $\begin{array}{l}\text { Heterogenous Goods, } \\
\text { Unit Demand }\end{array}$ & Demange et al. (1986) & Crawford and Knoer (1981) \\
\hline $\begin{array}{l}\text { Homogenous Goods, } \\
\text { Diminishing } \\
\text { Marginal Utility }\end{array}$ & Ausubel (2004) & Uniform-price auction \\
\hline $\begin{array}{l}\text { Heterogenous Goods, } \\
\text { Gross Substitutes }\end{array}$ & $\begin{array}{l}\text { Gul and Stacchetti } \\
(2000)\end{array}$ & Kelso and Crawford (1982) \\
\hline $\begin{array}{l}\text { Heterogenous Goods, } \\
\text { General Preferences }\end{array}$ & This paper & $\begin{array}{l}\text { Parkes (1999), } \\
\text { Ausubel and Milgrom (2002) }\end{array}$ \\
\hline
\end{tabular}

Table 1: This auction literature can be categorized based on the underlying algorithm.

subgradient algorithm requires the report of only one element of the demand correspondence. While both algorithms converge to an optimal solution, only the primal-dual algorithm is guaranteed to do so in a finite number of iterations. Hence there is a tradeoff in the merits of the two algorithms: complexity of each iteration versus number of iterations. For instance, since the auctions of Parkes (1999) and Ausubel and Milgrom (2002) are finite, they do not necessarily converge to the exact solution (though Parkes and Ungar (2002) provide upper bounds on this efficiency loss).

\section{The Efficient Allocation Problem}

There is a finite set of bidders $N$, and a finite set of indivisible objects (or goods), $G$. Each bidder $j \in N$ has a non-negative, integer valuation for each set of objects $H \subseteq G$ denoted $v_{j}(H) \in \mathbb{N}_{0}$ (with $\left.v_{j}(\emptyset)=0\right)$. We assume that $v(\cdot)$ is non-decreasing, i.e. $H \subseteq H^{\prime}$ implies $v_{j}(H) \leq v_{j}\left(H^{\prime}\right)$. Preferences are quasi-linear: A bidder $j$ who consumes $H \subseteq G$ and makes a payment of $p \in \mathbb{R}$ receives a net payoff of $v_{j}(H)-p$.

The set of object assignments is denoted as follows.

$$
\Gamma=\left\{\mu \in\left(2^{G}\right)^{|N|}: i \neq j \text { implies } \mu_{i} \cap \mu_{j}=\emptyset\right\}
$$

An assignment need not allocate all objects, since we do not require $\bigcup_{j \in N} \mu_{j}=$ 
$G$. Assignment $\mu \in \Gamma$ is efficient if it is one that maximizes $\sum v_{j}\left(\mu_{j}\right)$. Efficient assignments allocate all objects, due to the monotonicity assumption.

The problem of finding an efficient assignment can be solved in various ways. In order for a linear (as opposed to integer) program to solve such a problem, it is necessary for the program to have sufficient strength. ${ }^{4}$ We use the following linear programming formulation based on Bikhchandani and Ostroy (2002). Setting $\delta_{\mu}=1$ is interpreted as selecting the assignment $\mu$; setting $y_{j}(S)=1$ is interpreted as assigning bundle $S$ to bidder $j$. Dual variables are listed in parentheses.

$$
\begin{aligned}
& \max \sum_{j \in N} \sum_{S \subseteq G} v_{j}(S) y_{j}(S) \\
& \text { s.t. } y_{j}(S)-\sum_{\mu: \mu_{j}=S} \delta_{\mu}=0 \quad \forall j \in N, \forall S \subseteq G \quad\left(p_{j}(S)\right) \\
& \sum_{S \subseteq G} y_{j}(S)=1 \quad \forall j \in N \quad\left(\pi_{j}\right) \\
& \sum_{\mu \in \Gamma} \delta_{\mu}=1 \\
& 0 \leq y_{j}(S) \quad \forall S \subseteq G, \forall j \in N \\
& 0 \leq \delta_{\mu} \quad \forall \mu \in \Gamma
\end{aligned}
$$

Bikhchandani and Ostroy (2002) show that this formulation has an optimal integer solution, i.e. it finds an efficient assignment; this turns out to be a consequence of Corollary 1 below. While the second set of constraints is redundant, the corresponding dual variables $\pi_{j}$ can be interpreted as each bidder $j$ 's surplus (net payoff). The dual variable associated with the first type of constraint, $p_{j}(S)$, is interpreted as bidder $j$ 's price for the set $S$. The variable $\pi^{s}$ is interpreted as the seller's surplus.

Observe that the equality constraints could be replaced by inequality constraints $(\leq)$ without affecting the program's value. ${ }^{5}$ Since each $v_{j}(S)$ is non-negative, the first constraint can be made to hold with equality without decreasing the objective function. Since $v_{j}(\emptyset) \equiv 0$, it would not affect the objective function to add weight to $\delta_{\mu}$, where $\mu$ is the assignment that assigns all bidders the empty set; hence the third constraint also can be made

\footnotetext{
${ }^{4}$ Linear relaxations of some integer programs may not find integer feasible outcomes in some classes of problems; see Bikhchandani et al. (2002).

${ }^{5}$ We thank the Associate Editor for emphasizing this.
} 
to hold with equality without cost. Finally, the redundancy of the second constraint means it, too, can be made to hold with equality in an optimal solution. Inequality constraints would directly imply non-negativity of the dual variables. Nevertheless, we have chosen equality constraints in order to simplify some of the analysis below and to make it more similar to the canonical primal-dual algorithm described in Section 3.

The dual is

$$
\begin{aligned}
& \min \sum_{j \in N} \pi_{j}+\pi^{s} \\
& \text { s.t. } \pi_{j}+p_{j}(S) \geq v_{j}(S) \quad \forall j \in N, \forall S \subseteq G \quad\left(y_{j}(S)\right) \\
& \pi^{s}-\sum_{j \in N} p_{j}\left(\mu_{j}\right) \geq 0 \quad \forall \mu \in \Gamma
\end{aligned}
$$

The choice variables $p_{j}(S), \pi_{j}$, and $\pi^{s}$ are not sign-restricted. By the integrality of $(\mathbf{P})$ mentioned above, efficient assignments of objects can be supported by (dual) prices that are non-additive and non-anonymous (Section 4.3), meaning that different bidders see different prices for the same bundle.

\section{The Primal-Dual Auction}

We first give a brief overview of primal-dual algorithms. ${ }^{6}$ Consider a feasible program and its dual of the following form (with each $b_{j} \geq 0$ ). We omit obvious summation subscripts for brevity.

$$
\begin{aligned}
& \max \sum c_{i} x_{i} \\
& \text { s.t. } \sum a_{j i} x_{i}=b_{j} \quad \forall j \quad(\mathbf{p}) \\
& x_{i} \geq 0 \\
& \forall i \\
& \begin{aligned}
\min & \sum b_{j} \varphi_{j} \\
\text { s.t. } & \sum a_{j i} \varphi_{j} \geq c_{i} \quad \forall i
\end{aligned}
\end{aligned}
$$

If some $\varphi$ is an optimal solution to $(\mathbf{d})$, then there must exist a primal solution $x$ that satisfies the complementary slackness conditions: If $\sum a_{j i} \varphi_{j}>c_{i}$ then $x_{i}=0$. Letting $I=\left\{i: \sum a_{j i} \varphi_{j}>c_{i}\right\}$, this means the value of the following "restricted primal" (and its dual) must be zero.

\footnotetext{
${ }^{6}$ This description follows Chapter 5 of Papadimitriou and Steiglitz (1982).
} 


$$
\begin{array}{crcrr}
\max & \sum-z_{j} & \min \sum b_{j} \lambda_{j} & \\
\text { s.t. } & \sum a_{j i} x_{i}+z_{j}=b_{j} & \forall j & \text { s.t. } \sum a_{j i} \lambda_{j} \geq 0 & \forall i \notin I \\
& \forall i & (\mathbf{r p}) & \lambda_{j} \geq-1 & \forall j \quad(\mathbf{d r p}) \\
x_{i} \geq 0 & \forall j & & & \\
z_{j} \geq 0 & \forall i \in I & &
\end{array}
$$

Since $b \geq 0,(\mathbf{r p})$ is always feasible (e.g. $x=0$ and $z=b)$.

If instead $\varphi$ is not optimal, then these programs have a negative optimal value. In that case suppose $\lambda$ is a feasible solution for ( $\mathbf{d r p})$ that yields negative objective function value (not necessarily optimal). For any $\Delta>0$ this implies that $\sum b_{j}\left(\varphi_{j}+\Delta \lambda_{j}\right)<\sum b_{j} \varphi_{j}$, yielding an improved value to $(\mathbf{d})$. Of course to maintain feasibility in (d) requires $\Delta$ to satisfy $\sum a_{j i}\left(\varphi_{j}+\right.$ $\left.\Delta \lambda_{j}\right) \geq c_{i}$ for any constraint $i$. By choosing such a $\Delta$ and updating the dual variables from $\varphi$ to $\varphi+\Delta \lambda$ we obtain feasible dual variables that are "closer" to being optimal. The primal-dual algorithm repeats this adjustment until an optimal dual solution is reached. ${ }^{7}$

To implement this algorithm, there is some flexibility in choosing each iteration's direction of adjustment $\lambda$. Note that we did not require $\lambda$ to be an optimal solution to $(\mathbf{d r p})$ in the argument above. Any $\lambda$ that satisfies $\sum b_{j} \lambda_{j}<0$ would improve the dual variables as above. Therefore, we have some freedom in choosing $\lambda$. In fact, we use a modified version of (rp) omitting some of the $z_{j}$ 's - then choose $\lambda$ in a specific way (Theorem 1) to guarantee a monotonic adjustment of prices through each iteration.

In summary, the algorithm works as follows.

1. Choose a feasible solution to $(\mathbf{d})$.

2. Verify whether it is optimal by identifying a primal solution to (p) that is complementary to it. This is done by appending the complementary slackness conditions as constraints to $(\mathbf{p})$, yielding the "restricted primal" (rp).

3. If the dual solution was not optimal, then the dual (drp) tells you how

\footnotetext{
${ }^{7}$ Furthermore, the algorithm can do so in a finite number of steps if, for example, $\Delta$ is always chosen maximally (subject to each $\left.\sum a_{j i}\left(\varphi_{j}+\Delta \lambda_{j}\right) \geq c_{i}\right)$. Our proof of the finiteness of our implementation (Corollary 1 ) is even more straightforward.
} 
to adjust the dual solution to make it "closer" to optimal. Adjust the dual solution and repeat.

In Section 3.1 we perform these steps on our programs $(\mathbf{P})$ and $(\mathbf{D})$; subsequently we reinterpret these steps as an auction.

1. Choose low initial prices $p_{j}(S)$, e.g. $p_{j}(S) \equiv 0$.

2. Try to satisfy the bidders by finding an assignment which satisfies each bidder's demand at current prices.

3. If no such assignment exists, adjust prices and repeat.

One of our main points is to demonstrate that the construction of an ascending auction - determining how to adjust prices - can be performed by examining the primal-dual algorithm for the associated linear program.

\subsection{Implementing the Algorithm}

The choice variables of $(\mathbf{D})$ are of the form $p_{j}(S), \pi_{j}$, and $\pi^{s}$. To simplify matters, however, we are able to maintain the following equalities throughout the algorithm while ensuring feasibility.

$$
\begin{aligned}
\pi_{j} & =\max _{S \subseteq G}\left[v_{j}(S)-p_{j}(S)\right] \quad \forall j \in N \\
\pi^{s} & =\max _{\mu \in \Gamma} \sum_{j \in N} p_{j}\left(\mu_{j}\right) \\
p_{j}(\emptyset) & =0 \quad \forall j \in N
\end{aligned}
$$

It is simple to check that the first two conditions are naturally fulfilled by any optimal dual solution. To satisfy the third condition, we set each $p_{j}(\emptyset)=0$ as part of our initial, feasible dual solution, later ensuring that this value never changes (Definition 3).

Therefore, in our implementation of the primal-dual algorithm, we can refer to the $p_{j}(S)$ 's as the dual variables, since their choice determines the values of the remaining variables. Furthermore these equalities result in the economic interpretation of $\pi_{j}$ as bidder $j$ 's (potential) surplus at current prices, and of $\pi^{s}$ as that of the seller. 
Define, respectively, the set of active bidders, the demand correspondence of any bidder $j$, and the buyer-compatible part of the supply correspondence as follows.

$$
\begin{aligned}
& N^{+} \equiv\left\{j \in N: \pi_{j}>0\right\} \\
& D_{j} \equiv \arg \max _{S \subseteq G}\left[v_{j}(S)-p_{j}(S)\right]=\left\{S \subseteq G: \pi_{j}=v_{j}(S)-p_{j}(S)\right\} \\
& \Gamma^{*} \equiv\left\{\mu \in \Gamma: \pi^{s}=\sum_{j \in N} p_{j}\left(\mu_{j}\right), \text { and } \mu_{j} \in D_{j} \cup\{\emptyset\} \forall j \in N\right\}
\end{aligned}
$$

For simplicity of notation, we suppress the dependence of these concepts on the $p_{j}(S)$ 's. The set $\Gamma^{*}$ consists of the revenue maximizing allocations which are compatible with bidders' demand in the sense that a non-empty bundle is assigned only if it is demanded. Observe that, in general, this set may be empty. Given arbitrary prices $p_{j}(S)$, the assignment(s) that maximize seller revenue may allocate non-demanded sets of objects to the bidders. In our implementation of the algorithm below, however, $\Gamma^{*}$ remains non-empty due to the way we adjust variables.

The (non-redundant) complementary slackness (CS) conditions are as follows.

$$
\begin{aligned}
j \in N^{+} & \Longrightarrow y_{j}(\emptyset)=0 \text { and } \sum_{\emptyset \neq S \subseteq G} y_{j}(S)=1 \\
S \notin D_{j} & \Longrightarrow y_{j}(S)=0 \\
\pi^{s}-\sum_{j \in N} p_{j}\left(\mu_{j}\right)>0 & \Longrightarrow \delta_{\mu}=0
\end{aligned}
$$

These conditions have straightforward economic interpretation. Bidders who currently want something should receive something; non-demanded bundles should not be allocated; an assignment of objects should maximize seller revenue.

As described in the beginning of Section 3, our aim is to construct a restricted primal with respect to the constraints of $(\mathbf{P})$ and the complementary slackness conditions. To that end, if $(y, \delta)$ are feasible primal variables that satisfy (CS), then it is straightforward to check that $\delta_{\mu}=0$ for all $\mu \notin \Gamma^{*}$. By setting to zero and then removing all variables $\left(\delta_{\mu}\right)_{\mu \notin \Gamma^{*}}$ and $\left(y_{j}(S)\right)_{S \notin D_{j}}$, and appending the CS conditions to $(\mathbf{P})$, we obtain the following restricted primal, which we interpret below. We deviate slightly from the version of (rp) 
presented earlier in that we add artificial $\left(z_{j}\right)$ variables to only some of the primal constraints. (Upon a first reading, set $K=N^{+}$; later we explain our more general use of this program for $K \subseteq N^{+}$.)

$$
\begin{aligned}
& Z(K)=\max -\sum_{j \in K} z_{j} \\
& \text { s.t. } y_{j}(S)-\sum_{\mu \in \Gamma^{*}: \mu_{j}=S} \delta_{\mu}=0 \quad \forall j \in N, \forall S \in D_{j} \\
& \sum_{S \in D_{j}} y_{j}(S)+z_{j}=1 \quad \forall j \in N^{+} \\
& \sum_{S \in D_{j}} y_{j}(S)=1 \quad \forall j \notin N^{+} \\
& \sum_{\mu \in \Gamma^{*}} \delta_{\mu}=1 \\
& 0 \leq y_{j}(S) \quad \forall j \in N, \forall S \in D_{j} \\
& 0 \leq \delta_{\mu} \quad \forall \mu \in \Gamma^{*} \\
& 0 \leq z_{j} \quad \forall j \in N^{+}
\end{aligned}
$$

Since we have added artificial variables $\left(z_{j}\right.$ 's) only to some of the primal constraints instead of to all of the constraints from $(\mathbf{P})$ (thereby deviating from the standard prescription of the primal-dual method), it is conceivable that even $\left(\mathbf{R P}_{N^{+}}\right)$may be infeasible. We show (Theorem 2), however, that this never happens in our use of the program.

This program has a straightforward interpretation of which we make use below. For any $K \subseteq N^{+},\left(\mathbf{R P}_{K}\right)$ picks an assignment $\mu \in \Gamma^{*}$ to minimize the number of bidders in $K$ whose demands are left unsatisfied; $Z(K)$ is (the negative of) that number of unsatisfied bidders. It is worth noting that the artificial variables $z_{j}\left(j \in N^{+}\right)$correspond to the $y_{j}(\emptyset)$ variables in (P). Indeed, $\emptyset \in D_{j}$ if and only if $j \notin N^{+}$. Furthermore, if $z_{j}=1$ then $\sum_{S \in D_{j}} y_{j}(S)=0$, so $\delta_{\mu}=0$ whenever $\mu_{j} \in D_{j}$; therefore $\mu_{j}=\emptyset$ whenever $\delta_{\mu}>0$. It is to clearly highlight these as artificial variables that we do not write those constraints equivalently as $\sum_{S \in D_{j} \cup\{\emptyset\}} y_{j}(S)=1$.

As in the standard implementation of the primal-dual algorithm, if $Z\left(N^{+}\right)=$ 0 , then $(p, \pi)$ is an optimal dual solution and we are done. Otherwise a 
primal-dual algorithm adjusts the dual variables in accordance with the following dual.

$$
\begin{aligned}
Z(K)=\min \lambda^{s}+\sum_{j \in N} \lambda_{j} & \\
\text { s.t. } \lambda_{j}+\rho_{j}(S) \geq 0 & \forall j \in N, \forall S \in D_{j} \\
\lambda^{s}-\sum_{j \in N} \rho_{j}\left(\mu_{j}\right) \geq 0 & \forall \mu \in \Gamma^{*} \\
\lambda_{j} \geq-1 & \forall j \in K \\
\lambda_{j} \geq 0 & \forall j \in N^{+} \backslash K
\end{aligned}
$$

We interpret $\rho_{j}(S)$ as a (direction of) price change for bundle $S \in D_{j}$ for bidder $j$. As a consequence, we do not change the prices of non-demanded bundles. We interpret $\lambda_{j}$ as the change in bidder $j$ 's surplus and $\lambda^{s}$ as the change in the seller's surplus. When the program has a negative value, total (potential) surplus must decrease.

In order to define an ascending auction, we search for a solution to $\left(\mathbf{D R P}_{N^{+}}\right)$such that $\rho_{j}(S) \geq 0$ for all $j \in N$ and $S \in D_{j}$. Such a solution may not exist if the variables for $(\mathbf{D})$ - the $p_{j}(S)$ 's - are chosen arbitrarily (e.g. too high). It does exist, however, under a certain overdemand property defined below. That this property continues to hold throughout the adjustment procedure is what we intend to show.

Definition 1 Given the sets $N^{+}, D_{j}$, and $\Gamma^{*}$, we say that overdemand holds if $\left(\mathbf{R P}_{N^{+}}\right)$is feasible and $Z\left(N^{+}\right)<0$.

Feasibility of $\left(\mathbf{R P}_{N^{+}}\right)$requires $\Gamma^{*}$ to be nonempty. In turn, this implies that any unassigned object cannot be allocated in a way that creates additional revenue for the seller. In this sense, this rules out prices that get "too high."

The following definition is central to describing the price changes we use. We consider it to be the natural generalization of the concept of minimal overdemanded sets of objects introduced by Demange et al. (1986).

Definition 2 When overdemand holds, we say that a coalition $K \subseteq N^{+}$is undersupplied if $Z(K)<0$. Such a coalition $K$ is minimally undersupplied if for all $K^{\prime} \subsetneq K, Z\left(K^{\prime}\right)=0$. 
It is clear that the definition would not change if the phrase "all $K^{\prime} \subsetneq K$ " were replaced with "all $K^{\prime}=K \backslash\{j\}$ with $j \in K$." Furthermore, if overdemand holds then there exists at least one non-empty, minimally undersupplied coalition.

Our first main result is that non-negative price changes can be chosen so that only minimally undersupplied bidders see positive price increases.

Theorem 1 If overdemand holds then for any minimally undersupplied coalition $K \subseteq N^{+}$, there is a feasible solution to $\left(\boldsymbol{D} \boldsymbol{R} \boldsymbol{P}_{N^{+}}\right)$that yields negative value to $\left(\boldsymbol{D R} \boldsymbol{P}_{N^{+}}\right)$, and satisfies $\rho_{j}(S)=1$ for all $j \in K$ and $S \in D_{j}$, and $\rho_{j}(S)=0$ otherwise.

Proof Let $K$ be minimally undersupplied. By minimality, $Z(K \backslash j)=0$ for any $j \in K$; that is, the demands of $K \backslash j$ can be satisfied by some $\mu \in \Gamma^{*}$. Since $K$ is undersupplied, we have $Z(K)=-1$, i.e. precisely $|K|-1$ bidders in $K$ can be satisfied.

We construct an optimal solution to $\left(\mathbf{D R P}_{K}\right)$ where $\rho_{j}(S)=1$ for all $j \in K$ and $S \in D_{j}$, and $\rho_{j}(S)=0$ otherwise. Set $\lambda_{j}=-1$ for all $j \in K$ and $\lambda_{j}=0$ otherwise. Set $\lambda^{s}=|K|-1$. This is clearly feasible for $\left(\mathbf{D R P}_{K}\right)$ and is of value

$$
\lambda^{s}+\sum_{j \in N} \lambda_{j}=|K|-1-|K|=-1 .
$$

(By duality it is even optimal.) Since any feasible solution to $\left(\mathbf{D R P}_{K}\right)$ is feasible for $\left(\mathbf{D R P}_{N^{+}}\right)$, this completes the proof.

In fact, the conclusion of Theorem 1 also holds for any (possibly nonminimally) undersupplied $K \subseteq N^{+}$. We only make use of this result for minimally undersupplied sets of bidders in order to implement Vickrey-ClarkeGroves prices (Section 4).

Definition 3 In the proof of Theorem 1, we constructively derived the following price adjustment process.

1. Identify a minimally undersupplied set of bidders, $K \subseteq N^{+}$.

2. For each $j \in K$ and $S \in D_{j}$, add $\rho_{j}(S)=1$ to $p_{j}(S)$; otherwise do not change $p_{j}(S)$. (This preserves $p_{j}(\emptyset)=0$ for all $j$.)

3. For each $j \in K$, change $\pi_{j}$ by $\lambda_{j}=-1$; for each $j \notin K$ do not change $\pi_{j}$. 


\section{Increase $\pi^{s}$ by $\lambda^{s}=(|K|-1)$.}

Based on results below, we can interpret the repeated application of this price adjustment process as an ascending auction. In practice, steps 3 and 4 are carried out only implicitly in the execution of the auction. The fact that a bidder's surplus decreases by one (or zero) is a consequence of the price increases; a similar consequence exists for the seller. The steps are listed here for completeness in describing the exact primal-dual algorithm.

After such a price adjustment, it is clear that the demand correspondence for any bidder $j \notin K$ does not change. For $j \in K \subseteq N^{+}$, since valuations are assumed to be integral, a price increase of $\rho_{j}(S)=1$ for each $S \in$ $D_{j}$ can only enlarge $j$ 's demand correspondence; no demanded bundle can exit the demand correspondence (assuming integrality of prices throughout). ${ }^{8}$ Therefore, we have the following.

Lemma 1 If prices are integral and overdemand holds, then for every $j \in N$ the demand correspondence $D_{j}(\cdot)$ weakly increases after a price adjustment.

Conversely, if $p_{j}(S)$ has increased during any price adjustment, $S$ must be demanded by bidder $j$ at all future iterations. Therefore, if the algorithm is initialized at zero prices $(p \equiv 0)$, then only demanded bundles can have positive prices.

Another observation is that, after a price adjustment, the seller's demandcompatible supply correspondence $\Gamma^{*}$ can change in only two ways. First, some $\mu \in \Gamma^{*}$ could no longer be revenue maximizing after a price change. In this case, since the seller's revenue changes by $\lambda^{s}=(|K|-1)$, the change in revenue from $\mu$ must be $\left|\left\{j \in K: \mu_{j} \in D_{j}\right\}\right| \leq|K|-2$. Second, some $\mu \notin \Gamma^{*}$ could become revenue maximizing after a price change. This can happen only if $\left\{j \in K: \mu_{j} \in D_{j}\right\}=K$ and before the price change,

$$
\sum_{j \in N} p_{j}\left(\mu_{j}\right)=\sum_{j \in N} p_{j}\left(\mu_{j}^{\prime}\right)-1
$$

where $\mu^{\prime}$ is one of the assignments in $\Gamma^{*}$ that satisfies $|K|-1$ of the bidders in $K$.

To finally prove that prices increase throughout the algorithm, we have the following result.

\footnotetext{
${ }^{8}$ After the price adjustment, a demanded bundle may yield zero surplus to the bidder, but this bundle is still (weakly) demanded.
} 
Theorem 2 Beginning the algorithm at $p=0$, overdemand holds after each iteration of the price adjustment process until termination.

Proof It is clear that initially when each price is set to $p_{j}(S) \equiv 0$ overdemand holds: all bidders demand $D_{j}(p)=\{G\}$ and $\Gamma^{*}$ is the set of $n+1$ assignments where one bidder (or the seller) is assigned $G$ and all other bidders receive nothing. Clearly $\left(\mathbf{R P}_{K}\right)$ is feasible, $N^{+}=N$, and $Z(N)<0$.

Furthermore, if prices are integral at the beginning of an iteration, then they remain integral after the iteration, by our specification of unit price increases.

In order to prove the result, it suffices to prove that feasibility of $\left(\mathbf{R P}_{K}\right)$ holds from one iteration to the next; if $Z\left(N^{+}\right)<0$ after the iteration, then overdemand holds; otherwise, $Z\left(N^{+}\right)=0$ and the algorithm terminates with an optimal solution. Below, the subscript or superscript $t$ denotes the value of a variable during the $t^{\text {th }}$ iteration of the price adjustment.

Assume that $\left(\mathbf{R P}_{K^{t}}\right)$ is feasible during iteration $t$ (and that prices are integral). We show that an optimal solution to $\left(\mathbf{R P}_{K^{t}}\right)$ defines a feasible solution to $\left(\mathbf{R P}_{K^{t+1}}\right)$. Let $\left(\delta^{t}, y^{t}, z^{t}\right)$ be such an optimal solution, and without loss of generality suppose it is integral, ${ }^{9}$ so $\delta_{\hat{\mu}}^{t}=1$ for some $\hat{\mu}$. The assignment $\hat{\mu}$ satisfies the demand of $\left(\left|K^{t}\right|-1\right)$ bidders (where $K^{t}$ is the minimally undersupplied set in round $t)$. Since $\left(\pi^{s}\right)^{t+1}=\left(\pi^{s}\right)^{t}+\left(\left|K^{t}\right|-1\right)$, that assignment is still revenue maximizing for the seller in round $(t+1)$. With Lemma 1, this implies $\hat{\mu} \in \Gamma_{t+1}^{*}$.

Therefore, a feasible solution to $\left(\mathbf{R P}_{K^{t+1}}\right)$ can be obtained from $\left(\delta^{t}, y^{t}, z^{t}\right)$ by setting $\delta_{\hat{\mu}}^{t+1}=1$ and all other $\delta_{\mu}^{t+1}$ variables to zero. This implies $y_{j}^{t+1}\left(\hat{\mu}_{j}\right)=1$ for all $j \in N$ and $y_{j}^{t+1}(S)=0$ otherwise. The $z_{j}^{t+1}$ variables can obviously be chosen to complete a feasible solution; for all $j \in N_{t+1}^{+}, z_{j}=1$ if and only if $\hat{\mu}_{j}=\emptyset$.

Theorems 1 and 2 imply that prices are nondecreasing throughout the algorithm. Since the primal-dual algorithm eventually solves $(\mathbf{P})$, this defines an ascending auction that terminates with the efficient assignment.

Definition 4 A Primal-Dual (hereafter, PD) Auction is defined as an iterative procedure as follows.

1. Initially set $t=1$ and prices to zero: $p_{j}^{t}(S) \equiv 0$ for all $j \in N, S \subseteq G$.

\footnotetext{
${ }^{9}$ Integrality of $\left(\mathbf{R P}_{K}\right)$ follows from integrality of $(\mathbf{P})$.
} 
2. With respect to current round- $t$ prices, ask the bidders to reveal their demand correspondences, $D_{j}^{t}$.

3. If overdemand holds, perform the price adjustment process (Definition 3): choose a minimally undersupplied set of bidders $K^{t}$, and set $p_{j}^{t+1}(S)=p_{j}^{t}(S)+1$ for each $j \in K, S \in D_{j}^{t}$; all other prices remain the same $p_{j}^{t+1}(S)=p_{j}^{t}(S)$. Increase $t$ by one and return to Step 2 .

4. If overdemand does not hold, choose an assignment $\mu \in \Gamma_{t}^{*}$, and charge the bidders $p^{t}\left(\mu_{j}\right)$, ending the auction.

Observe that this definition does not uniquely define an algorithm, since (in Step 3) there may be multiple minimally undersupplied sets of bidders in a given round of the auction. ${ }^{10}$ In Section 4 we show (Theorem 4 ) that under some conditions this choice is inconsequential.

Corollary 1 Every PD Auction weakly increases all prices $p_{j}(S)$ until termination, yielding an efficient assignment in a finite number of steps.

This follows from Theorems 1 and 2. Since valuations are finite, there can only be a finite number of unit price increases.

\subsection{An Example}

In this section we present an example of a PD Auction applied to a setting with three bidders and three goods. Table 2 lists, for each round of the auction, the prices each bidder faces for each bundle, and the resulting surplus each bidder would obtain from each bundle at those prices. A bidder's demand correspondence is the set of bundles yielding maximum surplus (appearing as boldfaced surpluses in the table).

In each round, an undersupplied set of bidders is chosen. To choose such a set, the auctioneer must determine the set of revenue-maximizing assignments, $\Gamma^{*}$, which allocate objects in a demand-compatible way. The maximum revenue obtainable for the seller in each round is the seller's surplus, $\pi^{s}$, given in the last column of the table. Observe that in rounds one,

\footnotetext{
${ }^{10}$ This step is the main one distinguishing our auction from those of Parkes (1999) and Ausubel and Milgrom (2002). Their subgradient-based auctions prescribe price increases for all bidders that are not part of some provisional assignment of objects.
} 


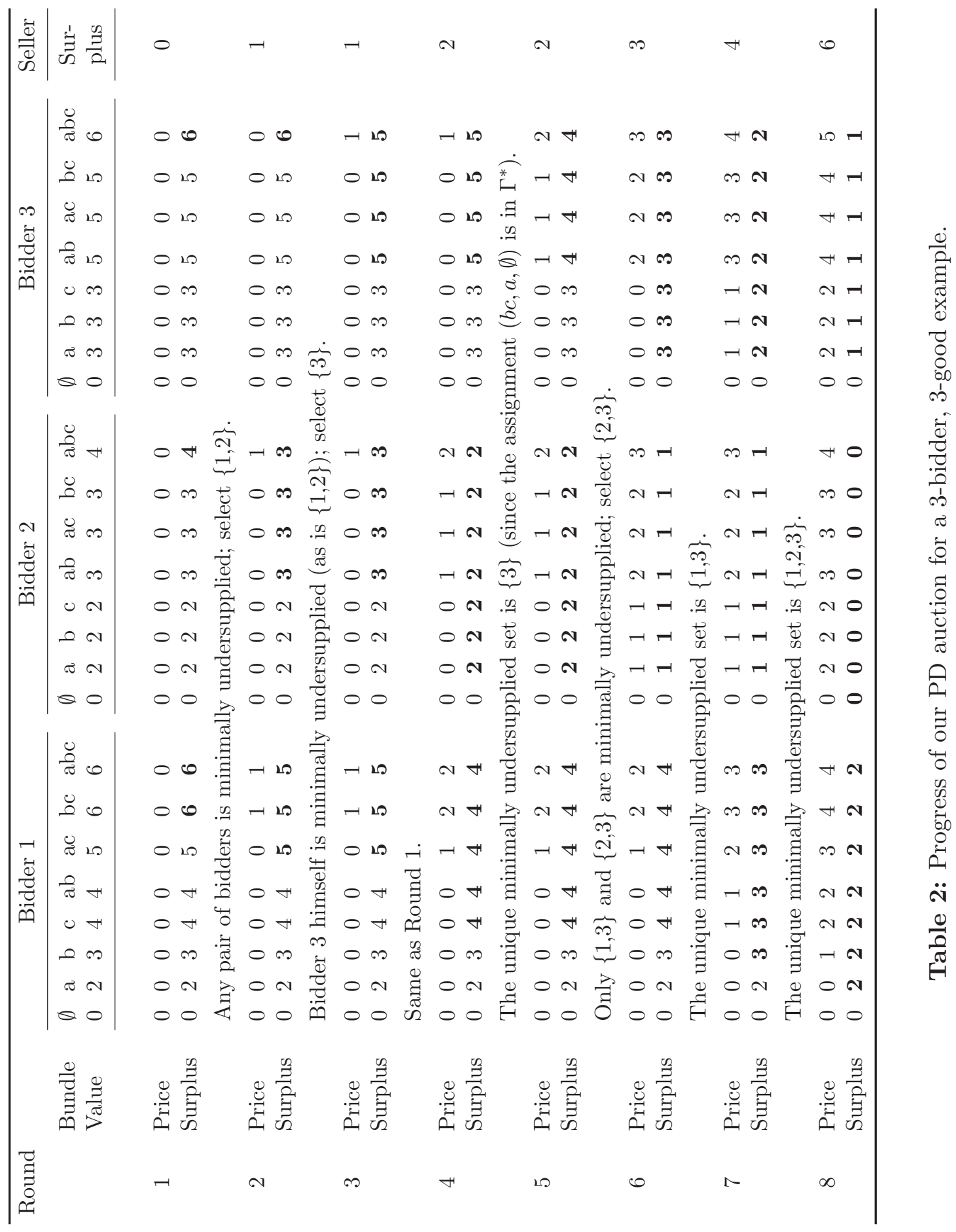


two, three, and five, there are multiple minimally undersupplied sets of bidders. For such rounds, the auctioneer must choose one of them, on whom he implements price increases. In Section 4 we discuss the implications of this choice.

For these valuation functions there are four efficient assignments in the example. One assigns (to Bidders 1,2 , and 3$)(c, b, a)$. The other three are $(c, \emptyset, a b),(c, a, b)$, and $(b c, \emptyset, a)$. Observe that each one is revenue maximizing and demand-compatible in the final round of the auction. Furthermore, each of them gives the bidders their Vickrey-Clarke-Groves payoffs (defined in Section 4).

\section{VCG Payments and Substitutability}

A well-known mechanism in the literature is the Vickrey-Clarke-Groves sealedbid auction. It chooses efficient outcomes based on the reported preferences of participating agents, and prescribes payments to/from the agents in a way that induces the agents to report their preferences truthfully. We formalize this mechanism in our environment with the following definitions.

Let $V(N)$ represent the value of $(\mathbf{P})$, which can be interpreted as the "social surplus" of the auction setting. More generally, for any subset of bidders $K \subseteq N$, let $V(K)=\max _{\mu \in \Gamma} \sum_{K} v_{j}\left(\mu_{j}\right)$. This amount would be the social surplus if only the bidders in $K$ were present.

With respect to a given efficient assignment $\mu^{*}$, bidder $j$ 's VCG payment is defined to be $V(N \backslash j)-\sum_{i \in N \backslash j} v_{i}\left(\mu_{i}^{*}\right)$. His resulting VCG payoff (or marginal product) is therefore $V(N)-V(N \backslash j)$. Since the bidder's reported valuations cannot affect the seller's calculation of $V(N \backslash j)$, he maximizes his payoff by reporting a valuation function which maximizes $V(N)$. This alignment of efficiency with a bidder's incentives is what makes the VCG payment scheme appealing.

In $(\mathbf{P})$, the dual variable associated with the constraint $\sum_{S \subseteq G} y_{j}(S)=$ 1 can be interpreted as bidder $j$ 's VCG payoff: Reducing the right hand side of this constraint to zero has the effect of removing bidder $j$ from the problem. The resulting change in the optimal objective function value is $V(N)-V(N \backslash j)$, which is bidder $j$ 's marginal product.

This argument shows only that among the set of optimal dual solutions to (D) there is one that gives bidder $j$ his marginal product. This argument does not prove that some optimal dual solution yields every bidder's 
marginal product simultaneously. In fact, there may not exist such a dual solution. Bikhchandani and Ostroy (2002) address this question by providing the following necessary and sufficient condition for such a dual solution to exist, simultaneously yielding all bidders' VCG payoffs (and hence their VCG payments).

Definition 5 We say that Agents are Substitutes when the marginal product of any set of bidders $M \subseteq N$ exceeds the sum of the marginal products of the individual bidders in $M$, i.e.

$$
V(N)-V(N \backslash M) \geq \sum_{j \in M}[V(N)-V(N \backslash j)] \quad \forall M \subseteq N
$$

This condition implies that individual bidders add relatively less to the social surplus than they do to the surplus of smaller coalitions. In addition, this condition is necessary and sufficient for the VCG payments (and the seller's VCG receipts) to be in the core of the cooperative game associated with this model. ${ }^{11}$ The VCG payoff scheme is in the core of such a game when, for any $M \subseteq N$, the total payoff to the bidders in $M$ plus the seller's VCG revenue weakly exceeds $V(M)$, i.e. when

$$
\sum_{j \in M}[V(N)-V(N \backslash j)]+\left[V(N)-\sum_{j \in N}[V(N)-V(N \backslash j)]\right] \geq V(M) .
$$

This is equivalent to (ASC).

Theorem 3 (Bikhchandani and Ostroy, 2002) If Agents are Substitutes (ASC), then among all optimal dual solutions $\left(\pi_{j}\right)_{j \in N}$ to $(\mathbf{D})$, the one that maximizes $\sum_{j \in N} \pi_{j}$ yields the bidders' $V C G$ payoffs: for all $j \in N$, we have $\pi_{j}=V(N)-V(N \backslash j)$.

Intuitively, to derive VCG payments with an ascending-price algorithm, this result leads us to decrease the values of $\pi_{j}$ in a "minimal" way, so as not to adjust beyond a dual solution which maximizes $\sum_{j \in N} \pi_{j}$. A PD auction does this by increasing prices only for minimally undersupplied sets of bidders.

\footnotetext{
${ }^{11}$ See Bikhchandani and Ostroy (2002). In the cooperative game, the set of agents is $N \cup\{s\}$ (where $s$ denotes the seller) and the characteristic function $\chi$ is defined so that for all $M \subseteq N, \chi(M \cup s)=V(M)$ and $\chi(M)=0$. A similar observation appears in Ausubel and Milgrom (2002).
} 
For this to work, though, requires that this type of substitutability condition hold also with respect to subsets of bidders. The reason for this is that, since the early rounds of a PD auction may force a bidder to compete against only a subset of other bidders (in a minimally undersupplied set), complementarities within that subset may drive prices too high. Intuitively, if the "wrong" subset of bidders is chosen to compete within itself, then prices on some bundles could be driven too high. In that case, VCG payments could not be reached monotonically.

Therefore, our main result in this section is that under the stronger condition of the submodularity of coalition values $V(\cdot)$, a PD auction yields VCG payments. ${ }^{12}$

Theorem 4 Suppose that for all $M \subseteq M^{\prime} \subseteq N$ and all $j \in N$ we have $V(M \cup\{j\})-V(M) \geq V\left(M^{\prime} \cup\{j\}\right)-V\left(M^{\prime}\right)$. Then any PD Auction terminates in VCG payments (regardless of the choice of minimally undersupplied set in each round).

Proof We show that at termination $\pi_{j}=V(N)-V(N \backslash j)$ for all $j \in$ $N$. Since the primal-dual algorithm terminates with optimal dual variables, it must terminate with $\pi_{j} \leq V(N)-V(N \backslash j)$ for all $j \in N$. This is because $V(N)-V(N \backslash j)$ is the effect of reducing the right-hand side of the corresponding primal constraint from one to zero, so $\pi_{j}$ cannot exceed that value.

Suppose by contradiction that by the monotonicity of the price adjustment process, there exists an iteration of the algorithm, $t$, such that (i) for all $j \in N, \pi_{j}^{t-1} \geq V(N)-V(N \backslash j)$ and (ii) for some $l \in N, \pi_{l}^{t}<V(N)-V(N \backslash l)$. By integrality, $\pi_{l}^{t-1}=\pi_{l}^{t}+1=V(N)-V(N \backslash l)$.

Since $l$ is part of the minimally undersupplied set selected in period $t-1$, $K^{t-1}$, there exists an assignment $\bar{\mu} \in \Gamma_{t-1}^{*}$ with $\bar{\mu}_{l}=\emptyset$ that is optimal for $\left(\mathbf{R P}_{K^{t-1}}\right)$. Let $M=\left\{j \in N: \bar{\mu}_{j} \neq \emptyset\right\}$, so $K^{t-1} \backslash\{l\} \subseteq M$. Let $\hat{\mu}$ be an assignment yielding value $V(M \cup\{l\})$.

Now,

$$
\sum_{j \in N: \bar{\mu}_{j} \neq \emptyset} p_{j}^{t}\left(\bar{\mu}_{j}\right)=\sum_{j \in N: \bar{\mu}_{j} \neq \emptyset} v_{j}\left(\bar{\mu}_{j}\right)-\pi_{j}^{t}
$$

\footnotetext{
${ }^{12}$ This submodularity condition is also the one under which Ausubel and Milgrom's (2002) auction implements VCG payments. See Section 5.
} 


$$
\begin{aligned}
& \leq V(M)-\sum_{j \in M} \pi_{j}^{t} \\
& <V(M)-\sum_{j \in M} \pi_{j}^{t}+\left(V(N)-V(N \backslash\{l\})-\pi_{l}^{t}\right) \\
& \leq V(M)-\sum_{j \in M \cup\{l\}} \pi_{j}^{t}+(V(M \cup\{l\})-V(M)) \\
& =V(M \cup\{l\})-\sum_{j \in M \cup\{l\}} \pi_{j}^{t} \\
& \leq \sum_{j \in N: \hat{\mu}_{j} \neq \emptyset} v_{j}\left(\hat{\mu}_{j}\right)-\sum_{j \in M \cup\{l\}} \pi_{j}^{t} \\
& \leq \sum_{j \in N: \hat{\mu}_{j} \neq \emptyset} p_{j}^{t}\left(\hat{\mu}_{j}\right)
\end{aligned}
$$

Analogous to the proof of Theorem 2 , since both $\bar{\mu} \in \Gamma_{t-1}^{*}$ and $\bar{\mu}$ was optimal to $\left(\mathbf{R P}_{K^{t-1}}\right)$, we know that $\bar{\mu} \in \Gamma_{t}^{*}$ as well.

Therefore at time $t$ the assignment $\hat{\mu}$ has better value to the seller than the seller optimal assignment $\bar{\mu}$, thereby providing a contradiction.

In the example of Section 3.2 (Table 2) the submodularity condition of Theorem 4 is satisfied, so the auction results in VCG payments. It is straightforward to observe that for those valuation functions, we have $V(\{1,2,3\})=V(\{1,3\})=9, V(\{2,3\})=7$, and $V(\{1,2\})=8$. Therefore, Bidder 2's VCG payoff is zero; he should either consume nothing, or pay his full value for whatever bundle he receives. This occurs in any of the four efficient assignments in the example. Similarly, the final surplus of Bidders 1 and 3 equal their VCG payoffs (of $V(\{1,2,3\})-V(\{2,3\})=2$ and $V(\{1,2,3\})-V(\{1,2\})=1$ respectively).

Without the submodularity condition in Theorem 4, a PD auction need not terminate in VCG payments. ${ }^{13}$ It is of interest to note, however, that even in such cases, a single (predetermined) bidder's VCG payment can be obtained by choosing, whenever possible, an undersupplied set excluding that bidder.

\footnotetext{
${ }^{13}$ Such an example is available upon request. The example shows that the Agents are Substitutes condition is not sufficient to guarantee that VCG payments result from any PD auction, depending on the choice of undersupplied sets of bidders.
} 
Theorem 5 Fix bidder $j$, and suppose that in each round, a PD Auction chooses a minimally undersupplied set of bidders $K$ so that, whenever possible, $j \notin K$. Then prices at termination give bidder $j$ his $V C G$ payoff.

Proof We show that by making such choices of minimally undersupplied sets, we have $\pi_{j}=V(N)-V(N \backslash j)$ at termination. Fixing $j$ as in the Theorem, let iteration $t$ be the last one at which $\pi_{j}^{t}=V(N)-V(N \backslash j)$. The existence of $t$ follows from the integrality of the price changes and valuations, as in the proof of Theorem 4 .

If the algorithm is finished then we are done. Otherwise, suppose in contradiction to the theorem that $\pi_{j}^{t+1}=V(N)-V(N \backslash j)-1$. This implies that in iteration $t$, bidder $j$ is in $N_{t}^{+}$(otherwise $j$ could not belong to $K^{t}$ ). By assumption, $j$ is a member of every minimally undersupplied set at iteration $t$, so $N_{t}^{+} \backslash\{j\}$ is not undersupplied (otherwise $N \backslash\{j\}$ would contain a minimally undersupplied set). Since $Z\left(N_{t}^{+} \backslash\{j\}\right)=0$ (in $\left(\mathbf{R P}_{K^{t}}\right)$ ), there exists $\mu \in \Gamma_{t}^{*}$ such that $\mu_{i} \in D_{i}^{t}$ for all $i \in N_{t}^{+} \backslash\{j\}$. Furthermore $\mu_{j}=\emptyset$, otherwise the algorithm would have finished in iteration $t$. For any remaining bidder $i \in N \backslash N_{t}^{+}$, observe that $\mu_{i} \in D_{i}^{t}$. (This follows from the observations that bidder $i$ does not drop out of $N_{t}^{+}$until each $p_{i}(S)=v_{i}(S)$, and that those prices stop increasing at that point. Hence $D_{i}$ is the powerset of $G$ whenever $i \in N \backslash N_{t}^{+}$.)

Since the (iteration $t$ ) dual variables are not optimal for $(\mathbf{D})$ we have

$$
\begin{aligned}
V(N) & <\left(\pi^{s}\right)^{t}+\sum_{i \in N} \pi_{i}^{t}=\sum_{i \in N \backslash j} p_{i}^{t}\left(\mu_{i}\right)+\sum_{i \in N} \pi_{i}^{t} \\
& =\sum_{i \in N \backslash j}\left[v_{i}\left(\mu_{i}\right)-\pi_{i}^{t}\right]+\sum_{i \in N} \pi_{i}^{t} \leq V(N \backslash j)+\pi_{j}^{t} \\
& =V(N \backslash j)+V(N)-V(N \backslash j)=V(N)
\end{aligned}
$$

which is a contradiction.

\subsection{Incentives}

When VCG payments are implemented through the use of a sealed-bid auction (i.e. a direct revelation mechanism), bidders maximize their payoffs by bidding truthfully (i.e. truthfully reporting their valuations). In other words, the VCG mechanism is strategyproof. Therefore it would not be surprising for an ascending auction (i.e. extensive form game) that implements VCG 
payments to inherit good incentives properties. In fact, the argument is typically made that if such a (extensive form) game implements VCG payments, then "truthful behavior" (suitably defined) must be an equilibrium of the game.

The argument, following the logic of the Revelation Principle, may be made as follows. Suppose a bidder behaves in a way which is consistent with some false valuation function (different from his true one). Further suppose that this causes him to receive objects and make payments corresponding to the VCG payoff for the false valuation. By strategy-proofness of the VCG (direct revelation) mechanism, the bidder cannot be better off than if he had behaved truthfully.

Unfortunately, this argument does not work if the bidder could behave in a way which is inconsistent with any valuation function. ${ }^{14}$ Second, it does not apply if the bidder behaves consistently with respect to a valuation function that does not yield a VCG payoff for that valuation. In our setting, for example, that could happen if the (ASC) condition fails.

The first of these problems can be fixed by specifying the rules of the auction in a way that bidders must behave consistently with some valuation function. Secondly, observe that (i) a PD auction must result in an efficient outcome (with respect to the inferred valuation functions), and (ii) a bidder pays at least his VCG payment at the final, efficient assignment. Together, these imply that if a PD auction would result in VCG payoffs under "truthful" bidding, then such bidding is an equilibrium.

Theorem 6 Suppose the rules of a PD auction are specified so that any feasible bidding behavior in the auction is equivalent to truthful demand revelation for some valuation function. ${ }^{15}$ If the bidders' valuation functions are such that VCG payoffs would result under truthful bidding (e.g. under the submodularity condition of Theorem 4), then truthful bidding is a Nash Equilibrium.

Proof Suppose other bidders with valuations $v_{-j}$ are bidding truthfully. When bidder $j$ bids truthfully with respect to $v_{j}$, he obtains his VCG payoff; denote the resulting assignment as $\mu$. When he bids as if his valuation

\footnotetext{
${ }^{14}$ Similar difficulties appear in the literature on extensive-form implementation. See Moore and Repullo (1988).

${ }^{15}$ One way this can be done is by strengthening step (2.) of Definition 4 to require both (i) $D_{j}^{t+1} \supseteq D_{j}^{t}$, and (ii) if $S \subset S^{\prime}$ and $S \in D_{j}^{t}$ then $S^{\prime} \in D_{j}^{t}$.
} 
function is $v_{j}^{\prime}$, denote the resulting assignment as $\mu^{\prime}$ and his resulting payment (for $\mu_{j}^{\prime}$ ) as $p_{j}^{\prime}$. Finally, let $\mu^{\prime \prime} \in \arg \max \sum_{i \neq j} v_{i}(\cdot)$ be an assignment achieving value $V(N \backslash j)$.

By the nature of the dual variable $\pi_{j}$, a PD auction yields a payoff no higher than the VCG payoff. Applied to $v_{j}^{\prime}$, this implies

$$
v_{j}^{\prime}\left(\mu_{j}^{\prime}\right)-p_{j}^{\prime} \leq v_{j}^{\prime}\left(\mu_{j}^{\prime}\right)+\sum_{i \in N \backslash\{j\}}\left[v_{i}\left(\mu_{i}^{\prime}\right)-v_{i}\left(\mu_{i}^{\prime \prime}\right)\right]
$$

By bidding truthfully, bidder $j$ (with valuation $v_{j}$ ) receives his VCG payoff, which is

$$
\begin{aligned}
V(N)-V(N \backslash j) & =\sum_{i \in N} v_{i}\left(\mu_{i}\right)-\sum_{i \in N \backslash\{j\}} v_{i}\left(\mu_{i}^{\prime \prime}\right) \\
& \geq \sum_{i \in N} v_{i}\left(\mu_{i}^{\prime}\right)-\sum_{i \in N \backslash\{j\}} v_{i}\left(\mu_{i}^{\prime \prime}\right) \\
& =v_{j}\left(\mu_{j}^{\prime}\right)+\sum_{i \in N \backslash\{j\}}\left[v_{i}\left(\mu_{i}^{\prime}\right)-v_{i}\left(\mu_{i}^{\prime \prime}\right)\right] \\
& \geq v_{j}\left(\mu_{j}^{\prime}\right)-p_{j}^{\prime} .
\end{aligned}
$$

The first inequality follows from the efficiency of $\mu$, while the last follows from (1). Hence bidding truthfully is (weakly) best.

\subsection{Necessity of Submodularity}

Theorem 4 shows that if a submodularity condition is satisfied, then there exists an ascending auction that implements VCG payments. Here we show that such a condition is somewhat necessary for the existence of such an auction, in the following sense.

If the submodularity condition of Theorem 4 fails, then at least one bidder's valuation function (say Bidder 1) does not satisfy the following gross substitutes condition of Kelso and Crawford (1982). ${ }^{16}$

Definition 6 The valuation function $v_{j}$ satisfies gross substitutes if, for all prices $p, p^{\prime} \in \mathbb{R}_{+}^{2^{G} \times N}$ such that

$$
p_{j}(S)=\sum_{g \in S} p_{j}(g) \leq p_{j}^{\prime}(S)=\sum_{g \in S} p_{j}^{\prime}(g) \quad \forall j \in N, S \subseteq G
$$

\footnotetext{
${ }^{16}$ See Theorem 11 of Ausubel and Milgrom (2002).
} 


\begin{tabular}{cccc}
\hline & \multicolumn{3}{c}{ Bundle } \\
\cline { 2 - 4 } Bidder & $\mathrm{a}$ & $\mathrm{b}$ & $\mathrm{ab}$ \\
\hline 1 & 4 & 6 & 16 \\
2 & 2 & 10 & 12 \\
3 & $8+\alpha$ & $2+\alpha$ & $8+\alpha$ \\
\hline
\end{tabular}

Table 3: An example violating gross substitutability.

(i.e. are "additive") and for all $S \in D_{j}(p)$, there exists $S^{\prime} \in D_{j}\left(p^{\prime}\right)$ such that $\left\{g \in S: p_{j}(g)=p_{j}^{\prime}(g)\right\} \subseteq S^{\prime}$.

For the 2-object case, the failure of this condition by $v_{1}(\cdot)$ simply means $v_{1}(a b)>v_{1}(a)+v_{1}(b)$. In this 2-object case, we show that there exists a domain of valuation functions for the other bidders (2 through $n$ ) on which no ascending auction (defined below) can always implement VCG payments. In fact, this result is true even if we require the other bidders' valuation functions to satisfy the gross substitutes condition.

Gul and Stacchetti (2000) prove a result of this type when auction mechanisms are required to assign prices which are "additive" and "anonymous" (as defined in Section 4.3). Even when all bidders' valuation functions are required to satisfy gross substitutes, they show that no such ascending auction can always yield VCG payments. Theorem 4 shows how their impossibility result can be overcome by allowing a richer set of prices. The result in this section shows the degree to which our positive result depends on substitutability.

The intuition behind the more general result below can be given with a simple example. For two objects $G=\{a, b\}$, suppose that three bidders have the valuations given in Table 3 . Restricting attention to the cases where $\alpha \in\{-1,0,1\}$, it is efficient to give $b$ to Bidder 2, and $a$ to Bidder 3. Their respective VCG payments are $8-\alpha$ and 6 (while Bidder 1 pays and receives nothing). Observe that the submodularity condition of Theorem 4 fails in this example. For the case of three bidders, this is equivalent to the failure of (ASC) (which happens with respect to $M=\{2,3\}$ ).

If an ascending auction uses only "real price information" to determine assignments and payments (as we define below), then it must do two things. First, it must conclude by offering good $a$ to Bidder 3 at a price of 6 . Second, it must determine the value of $\alpha$ in order to offer good $b$ to Bidder 2 at the 
correct price of $8-\alpha$. However, for the class of Bidder 3's valuation functions obtained by varying $\alpha \in\{-1,0,1\}$, the value of $\alpha$ cannot be inferred from his demand correspondence until Bidder 3 "demands" the empty set, which occurs only when his price for $a$ exceeds $8+\alpha>6$. This contradicts the fact that his price for that object ascends throughout the auction and ends at 6 .

This idea can be extended to any situation where one bidder has a valuation function over two objects that does not satisfy gross substitutes. To formalize this, we define an ascending auction by generalizing Gul and Stacchetti's definition to allow for non-additive, non-anonymous prices. However, we strengthen the definition by requiring (with condition (3) below) that prices seen during the auction must represent actual payments which could be made by the bidders.

Definition 7 A price path is a function $P:[0,1] \rightarrow \mathbb{R}^{2^{G} \times N}$. For each bundle of goods $H \subseteq G$, interpret $P_{i, H}(t)$ to be the price seen by bidder $i$ for bundle $H$, at "time" $t$. A price path is ascending if for all $H \subseteq G$ the function $P_{i, H}(t)$ is non-decreasing in $t$.

An ascending auction adjusts prices based only on the reported demands of the bidders. The following definition captures that idea.

Definition 8 An ascending auction assigns to each profile of bidder valuation functions $v \in \mathbb{R}_{+}^{2^{G} \times N}$ both an ascending price path $P^{v}$ and a final assignment $\mu^{v}$ satisfying the following two conditions. First, for all valuation profiles $v, v^{\prime} \in \mathbb{R}_{+}^{2^{G} \times N}$,

$$
\left[\forall t \in[0,1], \forall j \in N, D_{j}\left(P^{v}(t) ; v\right)=D_{j}\left(P^{v}(t) ; v^{\prime}\right)\right] \Longrightarrow\left[P^{v^{\prime}}=P^{v}\right]
$$

where $D_{j}(P ; v)$ is $j$ 's demand correspondence under prices $P$ when his valuations are $v_{j}$. That is, if a change to valuation functions $v^{\prime}$ does not change the reported demands of bidders, then it does not change the resulting price path. Information is revealed only through demand revelation in the auction. ${ }^{17}$ Second, the final assignment satisfies demand, i.e.

$$
\mu_{j}^{v} \in D_{j}\left(P^{v}(1) ; v\right) \quad \forall v \in \mathbb{R}_{+}^{2^{G} \times N}
$$

\footnotetext{
${ }^{17}$ Naturally, the empty set may be demanded, so information may be revealed when prices get "too high" for a bidder.
} 
An ascending auction assigns goods such that each bidder $j$ receives a bundle $H$ that is in his demand correspondence at prices $P(1)$, and charges that bidder $P_{j, H}(1)$. In this sense, we require prices in an auction not to be merely artificial constructs.

Unfortunately, when at least one bidder has a valuation function that does not satisfy the gross substitutes condition, an ascending auction cannot always implement VCG outcomes on some class of problems, as the next result shows.

Theorem 7 Suppose that there are two objects $G=\{a, b\}$ and that $|N| \geq 3$. Suppose one bidder's valuation function, say $v_{1}$, fails the gross substitutes condition. Then there exists a class of gross substitutes valuation functions for the other bidders, $\left(\mathcal{V}_{j}\right)_{j>1}$, such that no ascending auction yields VCG payments for each profile from $\left\{v_{1}\right\} \times \mathcal{V}_{2} \times \cdots \times \mathcal{V}_{n}$.

Proof To prove the result, suppose without loss of generality that Bidder 1's valuation function fails GS, and that $v_{1}(a) \equiv x \leq v_{1}(b) \equiv y$. Then $v_{1}(a b)=x+y+z$ where $z>0$ (failing GS).

Let Bidder 2 have fixed valuations such that $v_{2}(a)=0$ and $v_{2}(b)=$ $v_{2}(a b)=y+z$, so $\mathcal{V}_{2}$ is a singleton. ${ }^{18}$ For $\alpha \in(0, z]$, consider Bidder 3's valuations to be of the form $v_{3}(a)=v_{3}(b)=v_{3}(a b)=x+\alpha$; this defines the class $\mathcal{V}_{3}$. Ignore additional bidders (or assign them infinitesimal valuations).

Since $\alpha \in(0, z]$ and $x \leq y$, the efficient assignment gives good $b$ to Bidder 2 and gives good $a$ to Bidder 3. The VCG payment of Bidder 2 is $y+z-\alpha$, while that of Bidder 3 is $x$ (and Bidder 1 obviously pays nothing).

To yield VCG payments, the price path must finish at $t=1$ by yielding $P_{3, a}(1)=x$ for any $\alpha \in(0, z]$. Since $P_{3, a}(\cdot) \leq x$ throughout the auction (by monotonicity), Bidder 3 never demands the empty set. Therefore, his demand correspondence, $\arg \max _{S} v_{3}(S)-P_{3, S}$, is independent of $\alpha \in(0, z]$.

By the requirement (2) of an ascending auction, this means that the price paths on the domain $\left\{v_{1}\right\} \times\left\{v_{2}\right\} \times \mathcal{V}_{3}$ must be independent of $\alpha$. This contradicts the fact that it should always yield Bidder 2's VCG payment, which does vary with $\alpha$.

This result may appear to contradict the results of Ausubel (2000) and Parkes and Ungar (2002), who provide auction algorithms which always yield VCG payments. This apparent contradiction is resolved by observing that

\footnotetext{
${ }^{18} \mathrm{~A}$ similar proof exists with strictly monotonic valuation functions.
} 
the algorithms provided by those authors do not satisfy our definition of an ascending auction.

For example, with restrictions on the class of valuation functions, Ausubel (2000) makes clever use of the observation that any increase in prices results in a corresponding decrease in payoffs for the bidders. Specifically, suppose that an auctioneer could determine not only "market clearing" (Walrasian) prices for the set of bidders $N$, but also determine such (hypothetical) prices if the set of bidders were $N \backslash j$. Since bidder $j$ 's VCG payment equals the effect of his presence on the other bidders, this amount can be inferred by examining the difference between these two price vectors. Hence, VCG payments for all bidders could be calculated by determining $n+1$ Walrasian price vectors, i.e. by running $n+1$ Walrasian auctions. ${ }^{19}$

Such an algorithm does not, as stated, satisfy Definition 8. Our definition allows for only a single set of (bundle) prices. While other price structures (such as Ausubel's) can be mapped into prices of this form, the resulting prices may no longer be ascending. In this instance, since bidder $j$ 's final payment in Ausubel's auction is determined by the difference between two ascending vectors of parameters - the Walrasian prices and the hypothetical prices without bidder $j$ - his surplus could actually increase during the auction. In this sense, the auction cannot be described in terms of ascending bundle prices.

\subsection{Insufficiency of Anonymous Prices}

The prices presented to bidders in the PD auction are non-anonymous, meaning that different bidders may see different prices for the same bundle; formally, prices are anonymous if $p_{j}(S)=p_{i}(S)$ for all $i, j \in N$ and all $S \subseteq G$. Furthermore, the prices are non-additive, meaning that the price of a bundle is not necessary the sum of the prices of the objects in that bundle; formally, prices are additive if $p_{i}(S)=\sum_{g \in S} p_{i}(g)$ for all $i \in N$ and all $S \subseteq G$. One may wonder whether, in some environments, such rich (non-anonymous and non-additive) prices are overly complex in achieving VCG payments.

For instance, one of the simplest special cases of our model is the assignment problem, in which bidders' valuation functions are of the form

\footnotetext{
${ }^{19} \mathrm{~A}$ similar reasoning combined with Theorem 5 shows that the bidders' VCG payments could be obtained for any type of valuation functions by running $n$ (or fewer) iterations of PD auctions, the $j^{\text {th }}$ iteration omitting $j$ from the chosen undersupplied sets whenever possible.
} 
$v_{j}(S)=\max _{g \in S} v_{j}(g)$ for all $S \subseteq G$. For this class of problems, Demange et al. (1986) describe an auction that uses anonymous, additive prices, and yields VCG payments. On the other hand, Gul and Stacchetti (2000) show that even if bidders' valuation functions satisfy the gross substitutes condition in the current model, there exists no ascending VCG auction that uses anonymous, additive prices.

The anonymity of prices can only be achieved in settings in which the VCG outcome is envy-free, i.e. where each bidder $j$ would rather make his own VCG payment in exchange for his prescribed bundle than to make any other bidder $i$ 's VCG payment in exchange for $i$ 's prescribed bundle. It is commonly observed that in many environments, the VCG outcome is not envy-free, hence anonymous prices are not "rich" enough in those environments. $^{20}$

We leave it as an open question as to whether (or under what assumptions) non-anonymous but additive prices could be used in an ascending auction to implement VCG payments. ${ }^{21}$

\section{Subgradient Algorithms}

By writing the efficient-allocation problem as a linear program $(\mathbf{P})$, we obtain the definition of PD auctions as a by-product of one particular method of solving that linear program: the primal-dual algorithm. It is therefore natural to wonder whether other methods of solving linear programs may yield auctions, and how they may resemble PD auctions.

In this section, we argue that an application of another such methodsubgradient algorithms - yields the type of auction algorithm described by Parkes (1999), Parkes and Ungar (2002), and Ausubel and Milgrom (2002).

\footnotetext{
${ }^{20}$ One clear exception is the previously mentioned assignment problem. Pápai (2003) shows that the VCG outcome is envy-free when all bidders have superadditive valuation functions.

${ }^{21}$ Under a liberal interpretation of this question, one could say that Ausubel (2000) answers this question affirmatively. As discussed above, our interpretation of auction prices (Definition 8) does not admit such an auction format.
} 
One uses a subgradient algorithm to solve a linear program

$$
\begin{aligned}
& Z=\max \sum_{j=1}^{n} c_{j} x_{j} \\
& \text { s.t. } \sum_{j=1}^{n} a_{i j} x_{j} \leq b_{i} \quad \forall i \in\{1, \ldots, m\} \\
& x_{j} \geq 0 \quad \forall j \in\{1, \ldots, n\}
\end{aligned}
$$

by first relaxing the first $m^{\prime} \leq m$ constraints, yielding

$$
\begin{array}{rlr}
Z(\theta)=\max & \sum_{j=1}^{n} c_{j} x_{j}+\sum_{i=1}^{m^{\prime}} \theta_{i}\left(b_{i}-\sum_{j=1}^{n} a_{i j} x_{j}\right) & \\
\text { s.t. } \sum_{j=1}^{n} a_{i j} x_{j} \leq b_{i} & \forall i \in\left\{m^{\prime}+1, \ldots, m\right\} \\
& x_{j} \geq 0 & \forall j \in\{1, \ldots, n\} .
\end{array}
$$

The $\theta_{i}$ multipliers are Lagrange multipliers. By the duality theorem of linear programming, $Z=\min _{\theta \geq 0} Z(\theta)$. Finding $\theta^{*} \in \arg \min _{\theta \geq 0} Z(\theta)$ can be accomplished using the subgradient algorithm.

At iteration $t$, the current value of the Lagrange multiplier $\theta^{t}$ is adjusted by choosing a subgradient of $Z\left(\theta^{t}\right), s^{t}$, and setting $\theta^{t+1}=\theta^{t}+\Delta_{t} s^{t}$, where $\Delta_{t}$ is a positive "step size." In fact, if $x^{t}$ is an optimal solution at iteration $t$ (yielding $Z\left(\theta^{t}\right)$ ), then one subgradient is $s^{t}=A x^{t}-b$, so one uses

$$
\theta^{t+1}=\theta^{t}+\Delta_{t}\left(A x^{t}-b_{i}\right)
$$

If $\sum_{j} a_{i j} x_{j}^{t}>b_{i}$, then $\theta_{i}^{t+1}>\theta_{i}^{t}$, increasing the "penalty term" on this violated constraint. Similarly, if $\sum_{j} a_{i j} x_{j}^{t}<b_{i}$, then $\theta_{i}$ is decreased.

If step sizes are chosen appropriately, this procedure converges to an optimal solution. However, convergence of the subgradient algorithm can be very slow (Fisher, 1981). 
We apply this technique to a variant $\left(\mathbf{P}^{\prime}\right)$ of $(\mathbf{P})$

$$
\begin{aligned}
& \max \sum_{j \in N} \sum_{S \subseteq G} v_{j}(S) y_{j}(S) \\
& \text { s.t. } y_{j}(S) \leq \sum_{\mu: \mu_{j}=S} \delta_{\mu} \quad \forall j \in N, \forall S \subseteq G \\
& \sum_{S \subseteq G} y_{j}(S) \leq 1 \quad \forall j \in N \\
& \sum_{\mu \in \Gamma} \delta_{\mu} \leq 1 \\
& 0 \leq y_{j}(S) \quad \forall S \subseteq G, \forall j \in N \\
& 0 \leq \delta_{\mu} \quad \forall \mu \in \Gamma
\end{aligned}
$$

by relaxing the coupling constraints $y_{j}(S) \leq \sum_{\mu: \mu_{j}=S} \delta_{\mu}$. Let $\theta_{j}(S) \geq 0$ be the corresponding multipliers. We interpret $\theta_{j}(S)$ as the price of bundle $S$ for bidder $j$ (analogous to $p_{j}(S)$ in the previous analysis).

$$
\begin{aligned}
& V_{\theta}(N)=\max \sum_{j \in N} \sum_{S \subseteq G} v_{j}(S) y_{j}(S)-\sum_{j \in N} \sum_{S \subseteq G} \theta_{j}(S) y_{j}(S)+\sum_{\mu \in \Gamma} \delta_{\mu}\left[\sum_{j \in N} \theta_{j}\left(\mu_{j}\right)\right] \\
& \text { s.t. } \sum_{S \subseteq G} y_{j}(S) \leq 1 \quad \forall j \in N \\
& \sum_{\mu \in \Gamma} \delta_{\mu} \leq 1 \\
& \delta_{\mu} \geq 0 \quad \forall \mu \in \Gamma \\
& y_{j}(S) \geq 0 \quad \forall S \subseteq G, \forall j \in N
\end{aligned}
$$

After rewriting the objective function as

$$
V_{\theta}(N)=\max \sum_{j \in N} \sum_{S \subseteq G}\left[v_{j}(S)-\theta_{j}(S)\right] y_{j}(S)+\sum_{\mu \in \Gamma} \delta_{\mu}\left[\sum_{j \in N} \theta_{j}\left(\mu^{j}\right)\right]
$$

it becomes easy to see that for a given $\theta$, the solution to this problem can be found as follows.

1. Choose $\mu$ to maximize $\sum_{j \in N} \theta_{j}\left(\mu^{j}\right)$ and set $\delta_{\mu}=1$. In other words, choose the assignment of objects that maximizes the seller's revenue at current prices; denote it $\mu^{t}$. 
2. For each $j \in N$ choose $S^{j}$ to maximize $v_{j}(S)-\theta_{j}(S)$ and set $y_{j}\left(S^{j}\right)=$ 1. In other words, choose for each bidder a single, payoff-maximizing bundle at current prices.

If $S^{j}$ is assigned to bidder $j$ under $\mu^{t}$, then the corresponding constraint $y_{j}\left(S^{j}\right) \leq \sum_{\mu: \mu_{j}=S} \delta_{\mu}$ is satisfied with equality. In this case, equation (4) tells us there is no change in $\theta_{j}\left(S^{j}\right)$. In other words, if a bidder's utility maximizing bundle is in the selected seller-revenue maximizing assignment $\mu^{t}$, then there is no price change on this bundle.

If $S^{j}$ is not assigned to bidder $j$ under $\mu^{t}$, then the constraint $y_{j}\left(S^{j}\right) \leq$ $\sum_{\mu: \mu_{j}=S} \delta_{\mu}$ is violated. Furthermore, equation (4) increases the value of $\theta_{j}\left(S^{j}\right)$ by $\Delta_{t}>0$, i.e. if this utility-maximizing bundle is not in $\mu^{t}$, then it sees a

price increase. Furthermore, the constraint $y_{j}\left(\mu_{j}^{t}\right) \leq \sum_{\mu: \mu_{j}=\mu_{j}^{t}} \delta_{\mu}$ becomes $0<1$. In this case $\theta_{j}(S)$ is decreased by $\Delta_{t}$, i.e. a non-demanded bundle that is a part of $\mu^{t}$ sees a price decrease.

Due to the last observation, this algorithm as described does not yield an auction with ascending prices. A modification of this algorithm that carefully handles ties and omits such price decreases can be made to yield ascending prices and result in an efficient assignment. Such modifications appear in the auctions of Kelso and Crawford (1982), Parkes and Ungar (2002), and Ausubel and Milgrom (2002).

\section{Conclusion}

We have demonstrated that an ascending auction for heterogeneous objects can be constructed through re-interpretation of a primal-dual algorithm. Writing the efficient-assignment problem as a suitable linear program yields dual variables which can be interpreted as bundle prices and, in some cases, as bidders' Vickrey-Clarke-Groves payments (in the spirit of Bikhchandani and Ostroy, 2002). A primal-dual algorithm adjusts dual variables in order to satisfy complementary slackness; we interpret our application of this method as an adjustment of prices to satisfy bidder demand.

Therefore, in some cases, VCG payments can be implemented through such an ascending procedure - a Primal-Dual Auction. We argue that in other cases, no ascending auction can do so (Theorem 7).

The central concept used in the PD auction to adjust prices is that of minimally undersupplied sets of bidders. It can be considered a generaliza- 
tion of a concept used by Demange et al. (1986) in the special case of the assignment problem (allocating at most one object per bidder). Demange et al. call a set of objects $H \subseteq G$ "overdemanded" when $H$ contains fewer objects than the number of bidders who demand only those objects, i.e.

$$
\mid\left\{j \in N: S \subseteq H \text { for all } S \in D_{j}\right\}|>| H \mid .
$$

In each round of their auction, each bidder sees a price increase for each object in some minimally overdemanded set of objects.

Observe that if $H$ is minimally overdemanded in their sense, then $\{j \in$ $N: S \subseteq H$ for all $\left.S \in D_{j}\right\}$ is undersupplied and must contain a minimally undersupplied set of bidders. A PD auction would increase the price of each object in that set, but in a non-anonymous way: a bidder's price for an object increases only if he currently demands it. This non-anonymity is required in the more general setting since, in general, VCG payments cannot be supported by anonymous prices. For this reason, PD auctions (and the notion of undersupplied bidders) generalize the auction of Demange et al. (1986).

\section{References}

Ausubel, Lawrence M. (2000). An efficient dynamic auction for heterogeneous commodities. Manuscript, University of Maryland. http://www . ausubel.com/auction-papers/ efficient-dynamic-auction.pdf

Ausubel, Lawrence M. (2004). An efficient ascending-bid auction for multiple objects. The American Economic Review, 94(5):1452-1475.

Ausubel, Lawrence M. and Paul R. Milgrom (2002). Ascending auctions with package bidding. Frontiers of Theoretical Economics, 1(1):Article 1, $1-42$.

http://www . bepress.com/bejte/frontiers/vol1/iss1/art1

Bikhchandani, Sushil, Sven de Vries, James Schummer, and Rakesh V. Vohra (2002). Linear programming and Vickrey auctions. In Mathematics of the Internet: E-Auction and Markets (edited by Brenda Dietrich and Rakesh V. Vohra), volume 127 of The IMA Volumes in Mathematics and its Applications. Springer-Verlag, New York, NY, pages 75-116. 
Bikhchandani, Sushil and Joseph M. Ostroy (2002). The package assignment model. Journal of Economic Theory, 107(2):377-406.

Cramton, Peter (1998). Ascending auctions. European Economic Review, 42(3-5):745-756.

Crawford, Vincent P. and Elsie Marie Knoer (1981). Job matching with heterogeneous firms and workers. Econometrica, 49(2):437-450.

Demange, Gabrielle, David Gale, and Marilda Sotomayor (1986). Multi-item auctions. Journal of Political Economy, 94:863-872.

Fisher, Marshall L. (1981). The lagrangian relaxation method for solving integer programming problems. Management Science, 27(1):1-18.

Gul, Faruk and Ennio Stacchetti (2000). The English auction with differentiated commodities. Journal of Economic Theory, 92(1):66-95.

Kelso, Alexander S., Jr. and Vincent P. Crawford (1982). Job matching, coalition formation and gross substitutes. Econometrica, 50(6):1483-1504.

Moore, John and Rafael Repullo (1988). Subgame perfect implementation. Econometrica, 56(5):1191-1220.

Papadimitriou, Christos H. and Kenneth Steiglitz (1982). Combinatorial Optimization. Prentice-Hall, Inc., Englewood Cliffs, NJ.

Pápai, Szilvia (2003). Groves sealed bid auctions of heterogeneous objects with fair prices. Social Choice and Welfare, 20(3):371-385.

Parkes, David C. (1999). ibundle: An efficient ascending price bundle auction. In Proceedings ACM Conference on Electronic Commerce (EC-99), Denver, CO. pages 148-157.

Parkes, David C. and Lyle H. Ungar (2002). An ascending-price generalized Vickrey auction. Technical report, Division of Engineering and Applied Sciences, Harvard University. http://citeseer.nj.nec.com/parkes02ascendingprice.html 